\title{
Farmacopuntura utilizando os acupontos Hou Hai e Bai Hui em protocolos de
} sincronização de estro em cabras

[Pharmacopuncture using acupoints Hou Hai and Bai Hui in estrous synchronization protocols in goats]

\author{
R.C.S.A. Araújo ${ }^{1}$, L.P. Barbosa ${ }^{2, *}$, R.S. Souza ${ }^{3}$, A.L.A. Santana ${ }^{3,4}$, \\ I.M.B. Carneiro ${ }^{5}$, W.M. Machado ${ }^{5}$, R.L.A. Vieira ${ }^{5}$, R.D.L. Jesus ${ }^{5}$

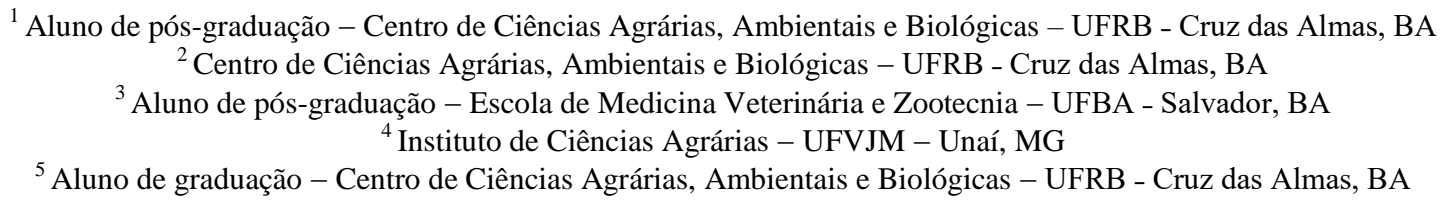

\section{RESUMO}

Avaliou-se a eficiência da administração de subdoses de eCG nos acupontos Bai Hui e Hou Hai em protocolos de sincronização de estro em cabras. Na primeira etapa, 57 cabras foram distribuídas aleatoriamente em quatro tratamentos: T1- 300UI de eCG intramuscular (IM); T2- 60UI de eCG no acuponto Hou Hai; T3- 60UI de eCG no acuponto Bai Hui e T4- 60UI de eCG IM; e na segunda etapa, 28 cabras foram distribuídas aleatoriamente em três tratamentos: T1- 300UI de eCG IM; T2- 30UI de eCG no acuponto Bai Hui e T3- 30UI de eCG IM. Ao final do tratamento hormonal, as cabras foram monitoradas para detecção do estro, realização das coberturas e avaliação do comportamento reprodutivo. Os dados foram submetidos à análise de normalidade, seguida dos testes estatísticos adequados para cada variável. Na primeira etapa experimental, obteve-se maior duração de estro nas cabras do T1 $(\mathrm{P}=0,009)$. $\mathrm{Na}$ segunda etapa experimental, obteve-se maior número de animais em estros no $\mathrm{T} 1(\mathrm{P}=0,03)$. As demais variáveis para ambas as etapas não sofreram influência dos tratamentos $(\mathrm{P}>0,05)$, demonstrando que a administração de subdoses de eCG nos acupontos Bai Hui e Hou Hai foi eficiente para sincronizar o estro.

Palavras-chave: ciclo estral, controle farmacológico, eCG, subdose

\begin{abstract}
The efficiency of administration of subdoses of eCG in the Bai Hui and Hou Hai acupoints in oestrus synchronization protocols in goats was evaluated. In the first stage, 57 goats were randomly assigned to four treatments: T1-300UI of intramuscular eCG (IM); T2- 60UI of eCG in acupoint Hou Hai; T3- 60UI of eCG in the Bai Hui acupoint and T4- 60UI of eCG IM; and in the second stage, 28 goats were randomly assigned to three treatments: T1-300UI of eCG IM; T2-30UI of eCG in the Bai Hui acupoint and T3- 30UI of eCG IM. At the end of the hormonal treatment the goats were monitored for estrus detection, and evaluation of reproductive behavior. The data were submitted to normality analysis, followed by appropriate statistical tests for each variable. In the first experimental stage, a longer duration of estrus in the T1 goats $(P=0.009)$ was obtained. In the second experimental stage, a greater number of animals were obtained in estrus at $T 1(P=0.03)$. The other variables for both experiments were not influenced by the treatments ( $P>0.05)$, demonstrating that administration of eCG subdoses in the Bai Hui and Hou Hai acupoints was efficient to synchronize the estrus.
\end{abstract}

Keywords: estral cycle, pharmacological control, eCG, subdose

Recebido em 3 de outubro de 2017

Aceito em 23 de agosto de 2018

*Autor para correspondência (corresponding author)

E-mail: larissa@ufrb.edu.br 


\section{INTRODUÇÃO}

Assim como em outros segmentos do agronegócio brasileiro, na caprinocultura existe demanda crescente pela aplicação de inovações tecnológicas que proporcionem melhorias na atividade (Teixeira et al., 2013). Para acelerar o crescimento da produtividade, aliado ao melhoramento genético, podem-se utilizar as biotécnicas da reprodução, como a sincronização do estro e da ovulação (Baldassare, 2007), associando, ainda, ao seu uso, a diminuição dos custos de produção (Uribe-Velásquez et al., 2010).

Alguns estudos têm sido realizados na busca pela dose mínima efetiva administrada por diferentes vias em protocolos hormonais para sincronização de estro em caprinos (Fonseca et al., 2005b; Souza, 2013). Segundo Luna et al. (2008), o uso de subdoses aplicadas em acupontos produz um efeito longo e similar à dose convencional, com a vantagem de causar menos efeitos colaterais.

Acuponto ou ponto de acupuntura, denominado Shu Xue na acupuntura tradicional chinesa, é uma região da pele onde há grande concentração de troncos nervosos, terminações neurais ou redes vasculares (Hwang e Egerbacher, 2006). Os acupontos Bai Hui e Hou Hai estão ligados aos órgãos reprodutivos, e alguns estudos estão sendo realizados com o uso desses acupontos como via de aplicação hormonal em protocolos de sincronização na espécie caprina (Souza, 2013; Araújo et al., 2014; Cardoso, 2014).

A técnica da acupuntura que se associa à sincronização de estro é a farmacopuntura, na qual se utiliza a aplicação de subdoses de fármacos em acupontos específicos, estimulando o acuponto por meio de pressão do tecido subcutâneo local, o que vai potencializar o efeito farmacológico (Souza, 2013). Dessa forma, a farmacopuntura torna-se uma alternativa para a administração hormonal em protocolos de sincronização de estro e ovulação.

O objetivo com este estudo foi avaliar a eficiência da administração de subdoses de gonadotrofina coriônica equina (eCG), nos acupontos Bai Hui e Hou Hai, em protocolos de sincronização de estro em cabras.

\section{MATERIAL E MÉTODOS}

O estudo foi realizado em duas etapas experimentais, sendo o projeto aprovado pelo Comitê de Ética no Uso de Animais (Ceua) da Universidade Federal do Recôncavo da Bahia (Protocolo no 23007.003070/2015-40).

A primeira etapa experimental foi executada na Fazenda Santana, no município de Iaçu-BA, situada a $12^{\circ} 46^{\prime}$ de latitude sul e $40^{\circ} 13^{\prime}$ de longitude oeste, localizada na região semiárida, clima quente, vegetação de caatinga, sem estações definidas, com precipitação média de $747 \mathrm{~mm}$ por ano. A temperatura média e a umidade relativa do ar no período experimental foram de $28^{\circ} \mathrm{C}$ e $73,4 \%$, respectivamente (Inmet, 2015).

Cinquenta e sete cabras da raça Anglo Nubiana foram selecionadas por meio de exame ginecológico, com avaliação ultrassonográfica por via transretal, apresentando condição corporal média de 2,35 $\pm 0,30$ e idade média de $2,95 \pm 0,10$ anos. Os animais foram submetidos a regime semi-intensivo de produção, com acesso à caatinga nativa e suplementação com silagem de sorgo e ração à base de farelo de milho, uma vez ao dia. Água e suplemento mineral foram fornecidos à vontade.

As cabras foram submetidas ao protocolo hormonal, inicialmente sob as mesmas condições. Receberam, durante o período de seis dias, esponjas intravaginais impregnadas com 60mg de acetato de medroxiprogesterona (MAP) (Progespon ${ }^{\circledR}$, Syntex, Buenos Aires, Argentina), acrescidas de $0,1 \mathrm{mg}$ de oxitetraciclina no momento do uso (Terra-Cortril ${ }^{\circledR}$ Spray, Pfizer, São Paulo, Brasil). Vinte e quatro horas antes da retirada das esponjas, foi administrado, via intramuscular, $0,125 \mathrm{mg}$ de cloprostenol sódico (Ciosin ${ }^{\circledR}$, Coopers, São Paulo, Brasil) em todas as cabras.

Após a retirada da esponja e a aplicação do agente luteolítico, os animais foram distribuídos em delineamento inteiramente ao acaso, em quatro tratamentos, sendo: T1 $(n=13)$ : aplicação intramuscular (IM) de 300UI de eCG (Novormon $^{\circledR}$, Coopers, São Paulo, Brasil) (100\% da dose); T2 (n= 14): aplicação de 60UI de eCG no acuponto Hou Hai (20\% da dose); T3 $(n=15)$ : aplicação de 60UI de eCG no acuponto Bai Hui 
(20\% da dose); e, T4 (n= 15): aplicação de 60UI de eCG em falso acuponto (IM) (20\% da dose).

Antes da aplicação hormonal, realizou-se tricotomia da área de aplicação do acuponto Bai Hui e antissepsia com iodopovidona (PVPi) (Riodeine $^{\circledR}$, Rioquímica, São Paulo, Brasil) na depressão localizada exatamente entre a distância média da base ventral da cauda e o ânus. Em seguida, com agulha hipodérmica $25 \times 8$, inserida no ângulo de $90^{\circ}$ perpendicular ao ponto de inserção, procedeu-se à injeção do fármaco.

A localização do acuponto Bai Hui está entre a última vértebra lombar e a primeira sacral, sendo a identificação do local realizada por palpação, e o agulhamento realizado acima do canal medular. Para aplicação nesse acuponto, foi utilizada agulha hipodérmica $25 \times 7$, inserida no ângulo de $90^{\circ}$ em relação à pele.

Após a retirada das esponjas e a aplicação da eCG, os animais foram avaliados a cada 12 horas para detecção do estro, com o auxílio de um reprodutor. Os parâmetros de comportamento reprodutivo avaliados foram: animais em estro (AE), intervalo entre a retirada da esponja e o início do estro (IREIE), intervalo entre a retirada da esponja e o final do estro (IREFE) e duração do estro (DE). Considerou-se como início do estro o momento em que a fêmea aceitou a monta e como final do estro quando esta deixou de aceitar a monta.

Para determinação da taxa de gestação e prolificidade, ao se detectar o estro, as cabras foram submetidas à monta natural, em intervalos de 12 horas. $\mathrm{O}$ número de coberturas variou em função da receptividade de cada fêmea ao macho reprodutor. $\mathrm{O}$ diagnóstico de gestação foi realizado 30 dias após as coberturas, mediante exame ultrassonográfico por via transretal (Pie Medical, modelo ÀquilaVet, acoplado a um transdutor linear de 6,0MHz). Após os nascimentos, determinou-se a prolificidade pela divisão do número de cabritos nascidos, vivos ou mortos, pelo número de partos.

Os dados foram submetidos à análise estatística. A normalidade dos erros experimentais entre os tratamentos para as variáveis foi avaliada previamente utilizando-se o teste de ShapiroWilk. As variáveis que não apresentaram distribuição normal (DE, IREIE e IREFE) foram avaliadas pelo teste de Kruskal-Wallis para verificar efeito de tratamento e, complementada essa análise, utilizou-se o teste de Mann-Whitney para comparar os escores médios entre os tratamentos. As variáveis binárias (taxa de gestação aos 30 dias - TG30d, AE) foram modeladas por meio da distribuição binomial. Para a variável prolificidade, a distribuição ajustada foi a de Poisson.

A segunda etapa experimental foi executada no Setor de Caprinocultura da Fazenda Experimental da Universidade Federal do Recôncavo da Bahia, no município de Cruz das Almas-BA, situado a $12^{\circ} 40^{\prime} 12^{\prime \prime}$ de latitude sul e 39 06' 07" de longitude oeste, cujo clima é quente e úmido. A temperatura média e a umidade relativa do ar no período experimental foram de $30^{\circ} \mathrm{C}$ e $89,2 \%$, respectivamente (Inmet, 2015).

Vinte e oito cabras da raça Anglo Nubiana foram selecionadas por meio de exame ginecológico, com avaliação ultrassonográfica por via transretal, apresentando condição corporal média

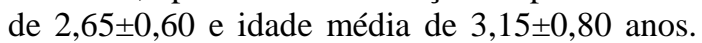
Os animais foram submetidos a regime semiintensivo de produção, com acesso ao pasto de capim-aruana (Panicum maximum) e suplementação com silagem de milho e ração à base de farelo de milho, uma vez ao dia. Água e suplemento mineral foram fornecidos à vontade.

O protocolo utilizado nessa etapa foi semelhante ao descrito para a primeira etapa experimental, variando apenas a dose de eCG e o acuponto utilizado, pois não se realizou aplicação no acuponto Hou Hai. Os animais foram distribuídos aleatoriamente em três tratamentos, sendo: T1 $(n=9)$ : aplicação intramuscular (IM) de 300UI de eCG (100\% da dose); T2 $(n=8)$ : aplicação de 30UI de eCG no acuponto Bai Hui (10\% da dose); e T3 (n= 10): aplicação de 30UI de eCG em falso acuponto (IM) (10\% da dose).

Os parâmetros de comportamento reprodutivo, diagnóstico de gestação e prolificidade foram avaliados conforme etapa experimental I, assim como a análise estatística dos dados.

\section{RESULTADOS E DISCUSSÃO}

Não houve diferença $(\mathrm{P}=0,09)$ para o percentual de cabras em estro, com média de $72,5 \%$ 
(Tab. 1), indicando que a via de aplicação, bem como a redução da dose de eCG em até $20 \%$ no protocolo de sincronização, não influencia na indução do estro. Fonseca et al. (2003) relataram que a resposta à indução estral em cabras pode variar de $60 \%$ a $100 \%$ e que fatores ambientais, individuais, de sanidade e nutricional exercem influência direta sobre esse parâmetro.
Considerando a variação na resposta à indução estral citada por esses autores, obteve-se no presente estudo taxa satisfatória para cabras em estro com a aplicação da dose reduzida de eCG no acuponto Bai Hui $(80,0 \%)$, entretanto a aplicação de $20 \%$ da dose em falso acuponto resultou em valor inferior $(53,3 \%)$ ao observado na literatura para a espécie caprina.

Tabela 1. Comportamento estral de cabras submetidas a protocolos de sincronização de estro utilizandose subdoses de gonadotrofina coriônica equina nos acupontos Bai Hui e Hou Hai

\begin{tabular}{lcccccc}
\hline $\begin{array}{l}\text { Parâmetro } \\
\text { s }\end{array}$ & P valor & 100\% IM & 20\% Hou Hai & 20\% Bai Hui & 20\% IM & Média \\
\hline AE (\%) & 0,09 & $92,3(12 / 13)$ & $64,3(9 / 14)$ & $80,0(12 / 15)$ & $53,3(8 / 15)$ & $72,5(41 / 57$ \\
IREIE (h) & 0,24 & $34,7 \pm 17,3$ & $22,4 \pm 24,2$ & $40,8 \pm 27,7$ & $29,8 \pm 30,8$ & $31,9 \pm 25,0$ \\
IREFE & 0,09 & $75,0 \pm 26,9$ & $44,3 \pm 40,7$ & $68,2 \pm 38,5$ & $43,4 \pm 44,5$ & $57,7 \pm 37,6$ \\
$(\mathrm{~h})^{2}$ & 0,009 & $40,3 \pm 18,9 \mathrm{a}$ & $21,9 \pm 23,3 \mathrm{ab}$ & $27,3 \pm 21,3 \mathrm{ab}$ & $13,6 \pm 15,0 \mathrm{~b}$ & $25,8 \pm 19,6$ \\
DE (h) & $2 *$ & &
\end{tabular}

$\mathrm{AE}=$ animais em estro; IREIE $=$ intervalo entre a retirada da esponja ao início do estro; IREFE $=$ intervalo entre a retirada da esponja ao final do estro; $\mathrm{DE}=$ duração do estro. $100 \% \mathrm{IM}=100 \%$ da dose de eCG por via intramuscular; $20 \%$ Hou Hai $=20 \%$ da dose de eCG no acuponto Hou Hai; $20 \%$ Bai Hui= 20\% da dose de eCG no acuponto Bai Hui e $20 \% \mathrm{IM}=20 \%$ da dose de eCG no falso acuponto. ${ }^{1}$ Dados paramétricos (média \pm desvio-padrão); ${ }^{2}$ Dados não paramétricos (mediana \pm intervalo interquartílico). *Letras diferentes na linha diferem entre si pelo teste de KruskalWallis a 5\% de significância.

Outros pesquisadores testaram a aplicação hormonal no acuponto Bai Hui em pequenos ruminantes e tiveram êxito em seus resultados. Martinez et al. (2007) testaram protocolo semelhante ao do presente estudo, porém avaliaram a eficácia apenas da aplicação de $20 \%$ da dose de PGF2 $\alpha(30 \mu \mathrm{g})$ no acuponto Bai Hui, e relataram que $66,7 \%$ das ovelhas apresentaram estro. Da mesma forma, Araújo et al. (2014) e Souza (2013) administraram 30\% da dose de eCG (90UI) e de PGF2 $\alpha(37,5 \mu \mathrm{g})$ no acuponto Bai Hui em cabras e obtiveram $94,3 \%$ e $100 \%$ de animais em estro, respectivamente. Nos dois últimos estudos, os valores encontrados foram semelhantes aos do controle (100\% da dose), mas também foram equivalentes aos encontrados com $30 \%$ da dose em falso acuponto, como observado no presente estudo, indicando que a dose utilizada para essas espécies muitas vezes está superestimada.

Para o intervalo da retirada da esponja ao início do estro (IREIE), obteve-se média de 31,9 horas, sem diferença entre os grupos $(\mathrm{P}=0,24)$ (Tab. 1). $\mathrm{O}$ intervalo obtido encontra-se dentro do esperado para a espécie caprina, que é de 12 a 48 horas após a retirada da fonte de progesterona, de acordo com Fonseca et al. (2005a) e Traldi et al. (2007). A média de IREIE obtida também é semelhante às encontradas por Maffili et al. (2006), utilizando 250UI de eCG em cabras (35,0 $\pm 5,89 \mathrm{~h})$, e por Nava-Trujillo et al. (2010), que encontraram um intervalo de $32,54 \pm 3,00 \mathrm{~h}$, utilizando 500UI desse hormônio. A avaliação desse parâmetro é importante para a identificação do momento da ovulação e deve ser levada em consideração para o estabelecimento do melhor momento de realização da IATF.

Donovan et al. (2000) relataram que baixos índices de fertilidade na IATF podem ser obtidos, principalmente, devido ao horário da ocorrência da ovulação após a sincronização do estro. Segundo Evans e Maxwell (1987), a determinação exata do momento da ovulação é crucial para o sucesso da inseminação, já que o oócito tem uma viabilidade curta, entre 12 e 24 horas.

O intervalo de retirada da esponja ao final do estro (IREFE) também não foi influenciado pelos tratamentos $(\mathrm{P}=0,09)$ e obteve-se média de 57,7 horas (Tab. 1). Apesar de não ter ocorrido influência dos tratamentos sobre os intervalos 
entre retirada de esponja e início e final de estro, a administração de $100 \%$ da dose de eCG por via intramuscular promoveu maior duração de estro em relação à aplicação de $20 \%$ da dose na mesma via, entretanto foi semelhante à aplicação de $20 \%$ da dose nos acupontos Hou Hai e Bai Hui $(\mathrm{P}=0,009)$ (Tab. 1). Tanto o grupo controle quanto o grupo tratado com $20 \%$ da dose no acuponto Bai Hui apresentaram duração de estro compatível com o padrão fisiológico para espécie caprina, que varia de 24 a 48 horas, de acordo com Maia e Bezerra (2010).

Segundo Fonseca et al. (2007), nos protocolos de indução e sincronização de estro, a gonadotrofina coriônica equina (eCG) promove o crescimento folicular, durante o qual os folículos produzem estrógeno, que, por sua vez, aumenta seus níveis no sangue e, em consequência, a duração do período de estro. Além disso, uma das teorias prováveis de mecanismo de ação da ativação do sistema reprodutor pela acupuntura, de acordo com Takada (2003), está relacionada com efeito direto dessa técnica no controle da esteroidogênese pela estimulação da produção e liberação de epinefrina e catecolaminas, por exemplo, as quais são estimulantes da esteroidogênese ovariana. Dessa forma, a acupuntura pode influenciar nas funções reprodutivas pelos sistemas autócrino e parácrino, bem como pela regulação do eixo hipotalâmico-hipofisário-gonadal. Com base nos resultados obtidos no presente estudo, sugere-se, portanto, que a subdose de 60UI de eCG $(20 \%$ Hou Hai e $20 \%$ Bai Hui) utilizada promoveu a elevação do estrógeno a níveis equivalentes à dose total de 300UI administrada na via IM (100\% IM), com respostas semelhantes na duração de estro.

Não houve influência dos tratamentos sobre a taxa de gestação aos 30 dias $(\mathrm{P}=0,67)$ (Tab. 2). Fonseca e Bruschi (2005) afirmaram que a taxa de gestação em caprinos pode variar entre 50 e $80 \%$ ao se utilizar monta natural ou inseminação artificial. Assim, apenas a taxa de gestação do grupo de cabras que receberam $20 \%$ da dose de eCG no acuponto Bai Hui está dentro do que é esperado para a espécie de acordo com a literatura. A semelhança entre os resultados, mesmo utilizando dose hormonal reduzida no protocolo de sincronização de estro, pode ser explicada pela utilização da técnica da acupuntura, haja vista que o estímulo de acupontos, associados à reprodução, altera os níveis plasmáticos de hormônios, como progesterona (Lin et al., 1998), produzida e secretada pelo corpo lúteo.

Tabela 2. Taxa de gestação aos 30 dias e prolificidade de cabras submetidas a protocolos de sincronização de estro utilizando subdoses de gonadotrofina coriônica equina nos acupontos Bai Hui e Hou Hai

\begin{tabular}{lcccccc}
\hline \multirow{2}{*}{ Parâmetros } & $\begin{array}{c}\text { P } \\
\text { valor }\end{array}$ & 100\% IM & 20\% Hou Hai & 20\% Bai Hui & 20\% IM & Média \\
\hline TG 30 (\%) & 0,67 & 46,2 & 42,8 & 60,0 & 20,0 & 42,2 \\
Prolificidade & 0,34 & $1,5 \pm 0,6$ & $1,3 \pm 0,5$ & $1,5 \pm 0,5$ & $1,5 \pm 0,7$ & $1,4 \pm 0,7$ \\
\hline
\end{tabular}

TG 30= taxa de gestação aos 30 dias; $100 \%$ IM=100\% da dose de eCG por via intramuscular; 20\% Hou Hai= 20\% da dose de eCG no acuponto Hou Hai; $20 \%$ Bai Hui $=20 \%$ da dose de eCG no acuponto Bai Hui e $20 \%$ IM= 20\% da dose de eCG no falso acuponto. Dados paramétricos (média \pm desvio-padrão).

Esse fato é comprovado pelo estudo de Souza (2013), o qual utilizou $30 \%$ da dose de eCG no acuponto Bai Hui em cabras e obteve $87,5 \%$ de gestação, entretanto relatou que, apesar de ter obtido taxa de gestação dentro do que se espera para a espécie, houve decréscimo no valor dessa variável quando aplicou a mesma dose $(30 \%$ de eCG) em falso acuponto, com 62,5 $\pm 0,5 \%$, demonstrando que a aplicação de subdoses hormonais sem estímulo a um ponto específico da reprodução pode reduzir o efeito esperado. Entretanto, Cardoso (2014), utilizando protocolo semelhante ao empregado neste trabalho, com $30 \%$ da dose de eCG no acuponto Hou Hai e falso acuponto, obteve $70,6 \%$ e $85,7 \%$, respectivamente, para taxa de gestação, sem diferenças entre os grupos.

A taxa média de gestação aos 30 dias para o grupo que recebeu a subdose (60UI) de eCG no acuponto Bai Hui foi de $60 \%$, superior ao observado por Maia Júnior et al. (2009), os quais trabalharam com cabras que utilizaram implante de progesterona por cinco dias, com aplicação de $50 \mu \mathrm{g}$ de PGF2 $\alpha$ e $250 \mathrm{UI}$ de eCG na retirada do dispositivo, e obtiveram 52,6\% de taxa gestação, o que sugere que a administração de uma dose reduzida de eCG é eficiente para promover 
melhores taxas de gestação, desde que seja administrada no acuponto Bai Hui, uma vez que a subdose no falso acuponto resultou em menor taxa de gestação.

Os valores médios obtidos neste estudo para prolificidade não diferiram entre os tratamentos $(\mathrm{P}=0,34) \quad($ Tab. 2), estando dentro da média encontrada para cabras adultas, que, segundo Simplício (2008), pode variar de 1,44 a 1,76 . Os valores foram semelhantes aos encontrados por Souza (2013), que relatou prolificidade média de $1,32 \pm 0,23$, ao utilizar a aplicação hormonal no acuponto Bai Hui. Cardoso (2014), quando fez uso de protocolo semelhante ao empregado neste trabalho, aplicou 30\% da eCG no acuponto Hou Hai e obteve 1,50 $\pm 0,55$ para prolificidade em cabras.
Houve diferença para o percentual de cabras em estro na segunda etapa experimental $(\mathrm{P}=0,03)$ (Tab. 3), na qual todas as cabras do grupo controle, que receberam $300 \mathrm{UI}$ ( $100 \%$ da dose) de eCG no momento da retirada da esponja intravaginal, apresentaram estro. A aplicação da subdose 30UI (10\% da dose) de eCG no acuponto Bai Hui promoveu resultado equivalente ao controle e foi suficiente para que houvesse manifestação de estro. Desse modo, pode-se sugerir que $10 \%$ da dose de eCG utilizada no acuponto Bai Hui foi suficiente para estimular o crescimento folicular, comprovando o que diz Lin et al. (1988) e Takada (2003) sobre os possíveis efeitos do uso de acupontos associados à reprodução.

Tabela 3. Comportamento estral de cabras submetidas a protocolos de sincronização de estro utilizando dose reduzida de gonadotrofina coriônica equina no acuponto Bai Hui

\begin{tabular}{lccccc} 
Parâmetros & P valor & $100 \%$ IM & $10 \%$ Bai Hui & $10 \%$ IM & Média \\
\hline AE$^{1}(\%) *$ & 0,03 & $100(9 / 9) \mathrm{a}$ & $55,5(5 / 9) \mathrm{ab}$ & $50,0(5 / 10) \mathrm{b}$ & $68,5(19 / 28)$ \\
IREIE $^{2}$ (h) & 0,13 & $44,2 \pm 14,8$ & $30,4 \pm 24,2$ & $21,0 \pm 25,9$ & $31,9 \pm 21,6$ \\
$\mathrm{IREFE}^{2}$ (h) & 0,40 & $72,2 \pm 11,2$ & $63,8 \pm 40,7$ & $42,7 \pm 45,9$ & $59,6 \pm 32,6$ \\
$\mathrm{DE}^{2}$ (h) & 0,46 & $27,9 \pm 12,3$ & $33,3 \pm 22,0$ & $31,1 \pm 24,2$ & $30,8 \pm 19,5$ \\
\hline
\end{tabular}

$\mathrm{AE}=$ animais em estro; IREIE=intervalo entre a retirada da esponja ao início do estro; IREFE=intervalo entre a retirada da esponja ao final do estro; $\mathrm{DE}=$ duração do estro. $100 \% \mathrm{IM}=100 \%$ da dose de eCG por via intramuscular; $10 \%$ Bai Hui $=10 \%$ da dose de eCG no acuponto Bai Hui e $10 \% \mathrm{IM}=10 \%$ da dose de eCG no falso acuponto. ${ }^{1}$ Dados paramétricos; ${ }^{2}$ Dados não paramétricos (mediana \pm intervalo interquartílico). *Letras diferentes na linha diferem entre si pelo teste de Kruskal-Wallis a 5\% de significância.

Araújo et al. (2014) não encontraram diferença ao aplicarem $30 \%$ das doses hormonais (PGF2 $\alpha$ e eCG) no acuponto Bai Hui e no falso acuponto, assim como Souza (2013)), que administraram $30 \%$ da dose de PGF2 $\alpha$ e eCG no acuponto Bai $H u i$ e em falso acuponto, em protocolo semelhante ao descrito neste trabalho, e obtiveram $100 \%$ e $75 \%$ de cabras em estro, respectivamente. Mesmo não ocorrendo diferença entre os grupos, os autores relatam que a maior reposta de estro no grupo que recebeu subdoses no acuponto Bai Hui aconteceu devido ao estímulo no ponto de acupuntura.

Os demais parâmetros de comportamento reprodutivo avaliados não apresentaram diferença entre os grupos (Tab. 3). O IREIE $(\mathrm{P}=0,13)$ apresentou média de 31,8 horas, com valores dentro do esperado para a espécie caprina (Traldi et al., 2007; Maia e Bezerra, 2010). O valor encontrado foi semelhante ao relatado por Rodrigues et al. (2015), que avaliaram o desempenho reprodutivo de cabras submetidas ao reuso do CIDR, associado à aplicação de $75 \mu \mathrm{g}$ de PGF2 $\alpha$ e à bioestimulação pelo efeito macho na retirada dos dispositivos, e obtiveram $31,1 \pm 11,27$ horas de intervalo entre a retirada da esponja e o início do estro.

O resultado encontrado por Araújo et al. (2014), ao utilizarem protocolo semelhante ao deste estudo, com administração de $30 \%$ de eCG (90UI) e $30 \%$ da dose de PGF2 $\alpha(37,5 \mu \mathrm{g})$ no acuponto Bai Hui, foi de 33,85 $\pm 23,90$ horas. Fonseca et al. (2005b) administraram 200UI de eCG na via intramuscular e $22,5 \mu \mathrm{g}$ de cloprostenol na via submucosa vulvar em cabras não lactantes e obtiveram $46,1 \pm 15,0 \mathrm{~h}$ e $53,6 \pm 16,1 \mathrm{~h}$ de intervalo para o início do estro após a retirada das esponjas nos protocolos de seis e nove dias, respectivamente. De acordo com esses autores, doses maiores de eCG promoveriam um aparecimento mais precoce do estro. Entretanto, esse fato não foi observado no 
presente estudo, uma vez que foram utilizados 30UI de eCG no acuponto Bai Hui e obteve-se menor intervalo em relação ao obtido por Fonseca et al. (2005b). Também Souza (2013), em seu trabalho com cabras, reduziu a dose de eCG em até $30 \%$ no acuponto Bai Hui e obteve intervalo de $63,4 \pm 23,2 \mathrm{~h}$, superior ao obtido no presente estudo.

O IREFE $(\mathrm{P}=0,40)$ apresentou média de 59,6 horas. Souza (2013) obteve maior intervalo em relação ao presente estudo, com média de $80,7 \pm 19,3 \mathrm{~h}$ ao administrar 90UI de eCG no acuponto Bai Hui, e também não encontrou diferença entre os grupos, assim como Araújo et al. (2014) e Cardoso (2014), que utilizaram as mesmas doses de eCG nos acupontos Bai Hui e Hou Hai e obtiveram médias de 80,7 $\pm 19,3$ h e $71,05 \pm 5,1 \mathrm{~h}$, respectivamente.

O estro durou, em média, 30,7 horas no presente estudo $(\mathrm{P}=0,46)$, resultado compatível com o padrão fisiológico para a espécie caprina, que varia de 24 a 48 horas, de acordo com Maia e Bezerra (2010). Souza (2013) e Araújo et al. (2014), ao utilizarem aplicação de doses reduzidas $(30 \%)$ de eCG e PGF2 $\alpha$ no acuponto Bai Hui, obtiveram média de 30,2h e 45,2h, respectivamente, assim como Cardoso (2014), quando empregou o mesmo protocolo de administração dos hormônios no acuponto $\mathrm{Hou}$ Hai, o qual relatou duração de 42,22h.

A gonadotrofina coriônica equina, quando utilizada nos protocolos de indução e sincronização de estro, além de promover elevação nos níveis de estrógeno por meio do estímulo da esteroidogênese e do crescimento de folículos no ovário, aumenta consequentemente a duração do período de estro (Fonseca et al., 2007). Essa afirmação, associada aos resultados obtidos neste estudo, sugere que a administração da subdose de 30UI de eCG (10\% Bai Hui e IM) promoveu elevação do estrógeno a níveis equivalentes à dose total de 300UI, administrado por via IM, o que foi observado na resposta semelhante de duração de estro.

A taxa de gestação aos 30 dias não apresentou diferença entre os grupos (Tab. 4) $(\mathrm{P}=0,56)$, com média de $40,3 \%$. A taxa de gestação obtida nos grupos $100 \%$ IM e $10 \%$ Bai Hui encontra-se dentro dos valores esperados para caprinos, que, segundo Fonseca e Bruschi (2005), pode variar de 50 a $80 \%$ para monta natural ou inseminação artificial, e próxima ao obtido por Pietroski et al. (2013), que relataram 60\% para monta natural.

Tabela 4. Taxa de gestação aos 30 dias e prolificidade de cabras submetidas a protocolos de sincronização de estro, que utilizaram dose reduzida de gonadotrofina coriônica equina no acuponto Bai Hui

\begin{tabular}{lccccc}
\hline Parâmetros & P valor & $100 \%$ IM & $10 \%$ Bai Hui & $10 \%$ IM & Média \\
\hline TG 30 (\%) & 0,56 & 55,5 & 55,5 & 10,0 & 40,3 \\
Prolificidade $^{\mathbf{1}}$ & 0,21 & $1,4 \pm 0,5$ & $2,2 \pm 1,1$ & $3,0 \pm 0,0$ & $2,2 \pm 0,8$
\end{tabular}

TG 30= taxa de gestação aos 30 dias; $100 \% \mathrm{IM}=100 \%$ da dose de eCG por via intramuscular; $10 \%$ Bai Hui= 10\% da dose de eCG no acuponto Bai Hui e $10 \% \mathrm{IM}=10 \%$ da dose de eCG no falso acuponto. ${ }^{1}$ Dados paramétricos (média \pm desvio-padrão). ${ }^{2}$ Dados não paramétricos.

O valor obtido para taxa de gestação, quando a eCG foi aplicada intramuscular ou no acuponto Bai Hui, corrobora o estudo realizado por Maia Júnior et al. (2009), os quais trabalharam com cabras que utilizaram cinco dias de implante de progesterona, com aplicação de $50 \mu \mathrm{g}$ de PGF $2 \alpha$ e 250UI de eCG na retirada do dispositivo, e obtiveram $52,6 \%$ de taxa de gestação, demonstrando a eficiência da aplicação de baixas doses de hormônio no acuponto Bai Hui. Souza (2013), ao testar $30 \%$ da dose de eCG no acuponto Bai Hui em cabras, obteve média de $75,0 \%$ de taxa de gestação e relatou não haver diferença entre os grupos controle e falso acuponto, assim como Cardoso (2014), que, ao utilizar subdoses no acuponto Hou Hai, obteve $77 \%$ de taxa de gestação.

Segundo Lin et al. (1988), a estimulação de acupontos associados com a reprodução altera significativamente os níveis plasmáticos dos hormônios luteinizante, folículo estimulante, estradiol e progesterona, e, de acordo com Acosta e Miyamoto (2004), a vascularização lútea é o principal fator responsável pela formação e manutenção da gestação devido à secreção de progesterona, o que pode explicar a semelhança entre os tratamentos para a taxa de gestação, mesmo se utilizando dose hormonal reduzida nos dois experimentos. 
Os valores obtidos para prolificidade foram equivalentes entre os grupos $(\mathrm{P}=0,21)$ (Tab. 4), com média de 2,2 nascidos. A prolificidade obtida nos grupos que receberam a subdose de eCG, independentemente do ponto de aplicação, foi superior ao esperado para a raça, que pode variar de 1,44 a 1,76, de acordo com Simplício (2008), e superior ao obtido no experimento 1, o que pode ser explicado pela diferença na condição corporal das fêmeas. Segundo Sarmento et al. (2010), a prolificidade é uma das características reprodutivas mais importantes para determinar a eficiência do sistema, pois está diretamente relacionada com a viabilidade econômica da exploração do rebanho. Esses autores indicaram, em seu estudo, que fêmeas leves apresentam baixa probabilidade de parir mais de uma cria, podendo esse número aumentar de acordo com o peso na cobrição, o que evidencia a importância da condição corporal no momento da concepção sobre o número de óvulos fecundados ou viabilizados em fetos. Os valores obtidos no presente estudo ao se administrarem $10 \%$ da dose no acuponto Bai Hui ou intramuscular, são superiores aos de Sarmento et al. (2010), que relataram prolificidade média do rebanho de 1,49 cabritos/matriz, ao estudarem o histórico de prolificidade de caprinos mestiços leiteiros com monta natural e sem a utilização de protocolos de sincronização de estro, no semiárido nordestino.

Resultados inferiores foram relatados por Souza (2013), que, ao aplicar 90UI no acuponto Bai Hui, obteve 1,25 $\pm 0,8$, e por Cardoso (2014), que, ao utilizar a mesma dose no acuponto Hou Hai,

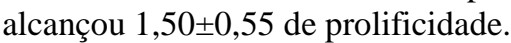

Os resultados obtidos corroboram os estudos de Luna et al. (2008), os quais sugerem que o uso de subdoses aplicadas em acupontos produz um efeito longo e similar à dose convencional, fato evidenciado no presente estudo por meio da manifestação de estro das cabras, bem como dos intervalos entre retirada da esponja e início e final de estro, duração de estro, taxa de gestação e prolificidade.

\section{CONCLUSÕES}

A administração de subdoses de eCG (20\% da dose tradicional) nos acupontos $\mathrm{Bai} \mathrm{Hui}$ e $\mathrm{Hou}$ Hai foi eficiente em sincronizar o estro de cabras, tratando-se, então, de vias alternativas de administração hormonal nessa espécie. A diminuição da dose de eCG para 60UI (20\%) em falso acuponto diminuiu a duração de estro, sendo necessária a adequação do horário de realização da IATF quando se reduz a quantidade desse hormônio. A utilização de subdoses de eCG, tanto de $20 \%$ como de $10 \%$ da dose tradicional, tendeu a promover uma diminuição na taxa de gestação e prolificidade quando foi utilizado o falso acuponto, tornando inviável a sua utilização nas doses de eCG testadas. Recomenda-se o uso de subdoses de eCG em até $10 \%$, aplicadas no acuponto Bai Hui, para sincronização de estro em cabras.

\section{REFERÊNCIAS}

ACOSTA, T.J.; MIYAMOTO, A. Vascular control of ovarian function: ovulation, corpus luteum formation and regression. Anim. Reprod. Sci., v.82, p.127-140, 2004.

ARAÚJO, R.C.A.; BARBOSA, L.P.; MACHADO, W.M. et al. Aplicação hormonal no acuponto Bai Hui em protocolos de sincronização de estro em caprinos. In: CONGRESSO NORDESTINO DE PRODUÇÃO ANIMAL, 9., 2014, Ilhéus. Anais... Ilhéus: [s.n.], 2014. p.252. (Resumo).

BALDASSARRE H. Reproducción asistida en la espécie caprina: inseminación artificial a clonación. Rev. Bras. Reprod. Anim., v.31, p.274-282, 2007.

CARDOSO, R.C. Farmacopuntura utilizando o acuponto hou hai em protocolos de sincronização de estro em caprinos. 2014. 73f. Dissertação (Mestrado em Ciência Animal) - Universidade Federal do Recôncavo da Bahia, Cruz das Almas, BA.

DONOVAN, A.; HANRAHAN, J.P.; DUFFY, P. et al. AI in sheep: breed differences in timing of ovulation. J. Agric. Food Res., v.39, p.3, 2000.

EVANS, G.; MAXWELL, W.M.C. Salamon's artificial insemination of sheep and goats. Butterworths, v.5, p.194, 1987.

FONSECA, J.F.; BRUSCHI, J.H. Reprodução assistida em pequenos ruminantes. Rev. Ciênc. Agrár., v.43, [13p.], 2005.

FONSECA, J.F.; BRUSCHI, J.H.; SANTOS, I.C.C. et $a l$. Induction of estrus in non-lactating dairy goats with different estrous synchrony protocols. Anim. Reprod. Sci., v.85, v.117-124, 2005b.

FONSECA, J.F.; SOUZA, J.M.G.; BRUSHI, J.H. Sincronização de estro e superovulação em ovinos e caprinos. In: SIMPÓSIO DE CAPRINOS E OVINOS DA ESCOLA DA V-ETERINÁRIA DA UNIVERSIDADE FEDERAL MINAS GERAIS, 2., 2007, Belo Horizonte. Anais... Belo Horizonte: [UFMG], 2007. p.167-194. (Palestra). 
FONSECA, J.F.; TORRES, C.A.A.; MAFFILI, V.V. et al. Desempenho reprodutivo de cabras Alpinas tratadas com hCG cinco dias após o acasalamento. Rev. Bras. Zootec., v.34, p.508-513, 2005a.

FONSECA, J.F.; TORRES, C.A.A.; MAFFILI, V.V.; PROSPERI, C.P. et al. Indução hormonal de estro em cabras nulíparas na estação de anestro. Rev. Bras. Reprod. Anim., v.27, v.528-530, 2003.

HWANG, Y.C.; EGERBACHER, M. Anatomia e classificação dos acupontos. In: SCHOEN, A.M. (Ed.). Acupuntura veterinária: da arte antiga à medicina moderna. São Paulo: Roca, 2006. p.17-23.

INMET, Instituto Nacional de Metereologia. Disponível em: <http://www.inmet.gov.br/portal/index.php?r=home/p age\&page $=$ rede_estacoes_conv_graf $>$. Acesso em: 29 de Julho de 2015.

LIN, J.H.; LIU, S.H.; CHAN, W.W.; WU, L.S.; PI, W.P. Effects of electroacupuncture and gonadotropinreleasing hormone treatments on hormonal changes in anoestrous sows. Am. J. Chin. Med., v.16, p.117-126, 1988.

LUNA, S.P.L.; ANGELI, A.L.; FERREIRA, C. et al. Comparison of pharmacopuncture, aquapuncture and acepromazine for sedation of horses. eCAM Advance Access published, v.5, p.267-272, 2008.

MAFFILI, V.V.; TORRES, C.A.A.; BRUSCHI, J.H. et al. Indução de estro em cabras da raça Toggenburg com dois diferentes dispositivos intravaginais. Arq. Bras. Med. Vet. Zootec., v.58, p.367-372, 2006.

MAIA JÚNIOR, A.; ARAÚJO, A.A.; SALLES, M.G.F. Indução e sincronização do estro e da ovulação em cabras leiteiras saanen com uso de dispositivos vaginais associados ou não à eCG ou efeito macho. Acta Vet. Bras., v.3, p.157-162, 2009.

MAIA, K.M.; BEZERRA, A.C.D.S. Controle do ciclo estral em caprinos: revisão. Acta Vet. Bras., v.4, p.419, 2010.

MARTINEZ, A.C.; OLIVEIRA, F.S.; CADAMURO, V.A.H. Uso de baixa dose de cloprostenol sódico administrado pelo ponto Bai Hui de acunpuntura em ovelhas. Arch. Vet. Sci., v.2, p.231-232, 2007.

NAVA-TRUJILLO, H.; CHANGO-VILLASMIL, J.; FINOL-PARRA, G. et al. Efecto de la dosis de eCG sobre la inducción del celo en cabras mestizas luego de un tratamiento corto con Medroxiprogesterona. Rev. Cient., v.20, p.181-183, 2010.
PIETROSKI, A.C.C.A.; BRANDÃO, F.Z; SOUZA, J.M.G. Short, medium or long-term hormonal treatments for induction of synchronized estrus and ovulation in Saanen goats during the nonbreeding season. Rev. Bras. Zootec., v.42, p.168-173, 2013.

RODRIGUES, I.C.S.; SALLES, M.G.F.; VIANA NETO, A.M. et al. Desempenho reprodutivo de cabras leiteiras submetidas à indução e sincronização do estro com o uso de dispositivos de progesterona reutilizados. Rev. Bras. Hig. Sanid. Anim., v.9, p.622633, 2015

SARMENTO, J.L.R.; PIMENTA FILHO, E.C.; ABREU, U.G.P. et al. Prolificidade de caprinos mestiços leiteiros no semiárido nordestino. Rev. Bras. Zootec., v.39, p.1471-1476, 2010.

SIMPLÍCIO, A.A. Estratégias de manejo reprodutivo como ferramenta para prolongar o período de oferta de carnes caprina e ovina no Brasil. Tecnol. Ciênc. Agropecu., v.2, p.29-39. 2008.

SOUZA, D.O. Sincronização de ovulação com redução de dose hormonal aplicada no acuponto Bai Hui em ovinos e caprinos. 2013. 87f. Dissertação (Mestrado em Ciência Animal) - Universidade Federal do Recôncavo da Bahia, Cruz das Almas, BA.

TAKADA, A. Uso da acupuntura na obstetrícia veterinária. 2003. Monografia (Curso de Especialização em Acupuntura Veterinária) Universidade Estadual Paulista "Júlio de Mesquita Filho", Botucatu.

TEIXEIRA, I.A.M.; GOMES, R.A.; CASTAGNINO, D.S. Inovações tecnológicas na caprinocultura. Rev. Bras. Saúde Prod. Anim., v.14, p.104-120, 2013.

TRALDI, A.S.; LOUREIRO, M.F.P.; CAPEZZUTO, A.; MAZORRA, A.L. Métodos de controle da atividade reprodutiva em caprinos. Rev. Bras. Reprod. Anim., v.31, p.254-260, 2007.

URIBE-VELÁSQUEZ， L.F.; SOUZA， M.I.L.; OSORIO, J.H. Resposta ovariana de cabras submetidas a implantes de progesterona seguidos de aplicações de gonadotrofina coriônica equina. Rev. Bras. Zootec., v.39, p.1214-1222, 2010. 